\title{
Non-Stationary Single-Channel Queuing System Features Research in Context of Number of Served Queries
}

\author{
Sergey Porshnev ${ }^{1}$ and Ivan Korelin ${ }^{1, *}$ \\ ${ }^{1}$ Ural Federal University, Institute of Radioelectronics and Information Technologies, 620002, 32 Mira street, Yekaterinburg, Russia
}

\begin{abstract}
This work devoted to researching of mathematical model of non-stationary queuing system (NQS). Arrival rate in studied NQS $\lambda(t)$ is similar to rate which observed in practice in a real access control system of objects of mass events. Dependence of number of serviced requests from time was calculated. It is proven that the ratio value of served requests at the beginning of event to all served requests described by a deterministic function, depending on the average service rate $\bar{\mu}$ and the maximum value of the arrival rate function $\lambda(\mathrm{t})$.
\end{abstract}

\section{Introduction}

The most of the known analytical solutions considered in the theory of queuing systems (QS) are obtained with assumption that QS is stationary and the input of requests is Poisson flow[1]. In addition, there are analytic solutions for:

1. QS with Erlang flow of requests (QS with limited aftereffect [5]).

2. QS with the flow of requests, representing a Markov process with a finite or countable state space [6].

3. QS with the flow of requests, rate of which is a semiMarkov process with discrete state space, (MC-flows or imbedded Markov chains) [2].

There is substantially less analytical solutions for the QS receiving random input flow of requests with rate $\lambda$, depending from the time of $\lambda=\lambda(t)$. Moreover, this class includes a large number of really existing QS, such as turnstiles, providing passages to the objects of mass events [4], passenger monitoring device in airports and train stations, etc.

The results of research of this class of QS can be used as a scientific basis for the making decision at the design stage for design and modernization of this type QS. In this regard, the study of this class of QS it is an urgent task for today from both scientific and practical points of view.

However, for instance, the issue about possibility of describing NQS characteristics, which were chosen as the research object, using deterministic functions which link the measured practical characteristics flow of requests (visitors) and the average serving rate of the operational device is opened.

In this paper are presented the results of a research of the NQS using the statistical modeling. The analysis of this results allowed us to offer analytical formulas describing the dependence of the NQS characteristics from maximum input flow rate $\lambda=\lambda(t)$ and the average service rate of the operational devise $\bar{\mu}$.

\section{Mathematical model of non-stationary queuing system}

Block diagram of a non-stationary model of singlechannel QS with unlimited queue is shown in Figure 1. Queue-discipline is first in first out (FIFO).

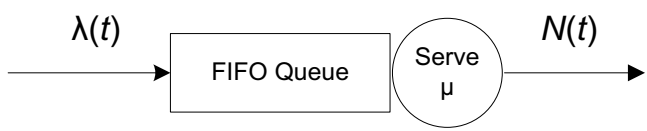

Fig. 1. The scheme of non-stationary QS model

This scheme shows that in this model QS input request flow with rate $\lambda=\lambda(t)$ is changing in time. Speed of service of incoming requests is determined by service rate, which is a random variable with probability density:

$p\{\xi\}=\left\{\begin{array}{c}0, \text { then } \xi<1, \\ \frac{2}{9(M[\xi]-1)}(\xi-1), \text { then } 1 \leq \xi<M[\xi], \\ \frac{2}{9(M[\xi]-10)}(\xi-10), \text { then } M[\xi]<\xi \leq 10, \\ 0, \text { then } \xi>10,\end{array}\right.$

where $\xi \in[1,10]$. The used probability density provides, that $\bar{\mu} \in(6,60)$.

Selection of arrival rate dependence from time type based on statistical analysis of real data, carried out in

* Corresponding author: korelin.ivan@gmail.com

(C) The Authors, published by EDP Sciences. This is an open access article distributed under the terms of the Creative Commons Attribution License 4.0 (http://creativecommons.org/licenses/by/4.0/). 
[4]. Typical dependence $\lambda_{\exp }(t)$ which was obtained in the football match between football clubs "Ural" and "Anji" at the Central Stadium (CS) of Ekaterinburg 27.09.2012 shown in Figure 2.

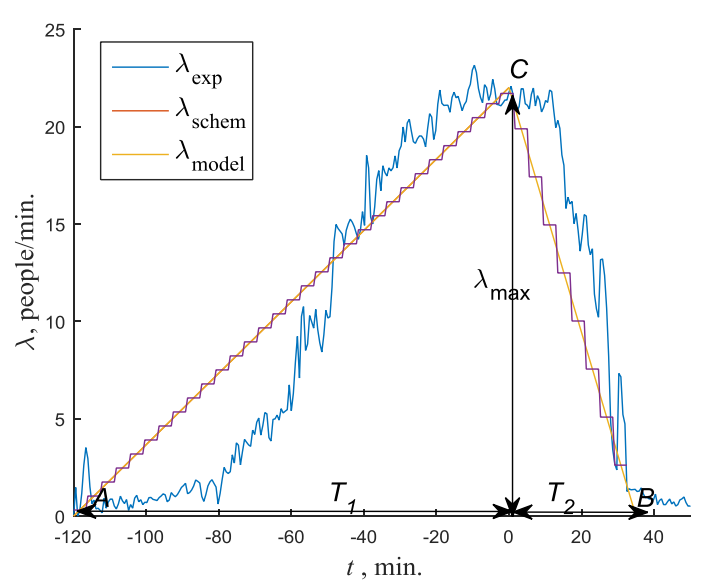

Fig. 2. Dependencies $\lambda(t)$ non-stationary queuing system (the beginning of the match, $t=0$ )

Figure 2 shows that access to CS was opened for 2 hours prior to the football game. In the considered NQS after the opening of the turnstiles during time $T_{1}=120$ minutes the arrival rate of requests (visitors) was growing from 0 people $/ \mathrm{min}$ to $\lambda_{\max }=22$ people $/ \mathrm{min}$. After the beginning of match during the time interval $T_{2}=30$ minutes arrival rate or requests (visitors) has decreased from $\lambda_{\max }=22$ people $/ \mathrm{min}$ to 0 people $/ \mathrm{min}$. Thus, mean number of persons passed in the CS through a single turnstile amounted to 1700 people.

$$
N=\int_{-120}^{30} \lambda(t) d t
$$

Piecewise-linear law depending on $\lambda(t)$ was used in the simulation because the main objective of the research was to study opportunity of describing of non-stationary queuing systems using deterministic functions. On the time interval $T_{1}[-120 ; 0] \min \lambda(t)$ increases linearly from 0 to $\lambda_{\max }$, further time interval $T_{2}[0,30]$ min decreased linearly from 0 to $\lambda_{\max }$. At the same time, $\lambda_{\max }$ is chosen so that the total number of people served in NQS is 1700. Piecewise constant approximation was used in carrying out statistical tests for the chosen law $\lambda_{\text {schem }}(t)$

Following [3], chosen approximation interval duration is equal to 30 seconds and the rate value for the corresponding interval was as average rate for this range.

\section{The methodology of the computational experiments}

The diagram of algorithm used for statistical modelling is described in detail in [3]. During statistical tests, parameter $T_{2}=30 \mathrm{~min}$ was fixed and parameters $T_{1}, \lambda_{\max }$ varied so that the total number of requests (visitors) $N$ was equal to 1700 (Fig. 3). It is enough to change one of the parameters, for example $T_{1}$, because for fixed $N$ and
$T_{2}$ parameters $T_{1}, \lambda_{\max }$ are linearly independent from each other:

$$
\lambda_{\max }=2 \cdot N /\left(T_{1}+T_{2}\right)
$$

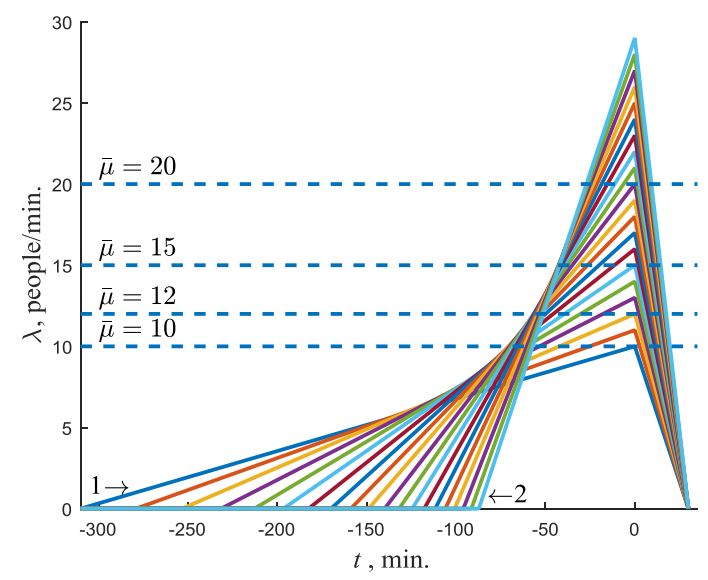

Fig. 3. Dependencies arrival requests rate from time with different values $\lambda_{\max }$ and $T_{1}$

In conducted studies parameter $T_{1}$ was varied in the range $[-310 ;-87]$ minutes and the value $\lambda_{\max }$ varied in the range of $[10,29]$ people/min. (Plots 1 and 2 in Figure 3, respectively). Analysis of the characteristics used for describing the features of the operation of studied NQS [3] showed that for their quantitative description can be used, for example, the dependence of the number of visitors entered to the stadium (in terms of QS - number of serviced requests) from time:

$$
N=N\left(\lambda_{\max }, \bar{\mu}, t_{k}\right)
$$

where $t_{k}=T_{1}+\frac{T_{2}-T_{1}}{K}(k-1), k=\overline{1, K}, K-$ the number of intervals of the piecewise linear approximation $\lambda(t)$. In the simulation, Monte Carlo method was used. It had average values of function $N=N\left(\lambda_{\max }, \bar{\mu}, t_{k}\right)$ with over the ensemble of realizations of independent values:

$$
\bar{N}\left(\lambda_{\max }, \bar{\mu}, t_{k}\right)=\frac{1}{m} \sum_{j=1}^{m} N_{j}\left(\lambda_{\max }, \bar{\mu}, t_{k}\right),
$$

where $m$ - the number of independent trials in the Monte-Carlo method.

\section{Analysis of the experimental results}

The results of calculations of the functions $\bar{N}\left(T_{1}, T_{2}, \lambda_{\max }, \bar{\mu}, t_{k}\right) \quad$ are showed below. During simulation the number of intervals of the piecewise linear approximation of the dependence $\lambda(t) K$ was equal to 680 . The number $m$ of independent statistical tests was 1000 . Dependencies $\bar{N}=\bar{N}\left(T_{1}, T_{2}, \lambda_{\max }, \bar{\mu}, t_{k}\right)$ for $\lambda_{\max } \in[10,29]$ people/min, $T_{1} \in[-310 ;-87] \mathrm{min}$, $T_{2}=30$ min., $\bar{\mu} \in\{10 ; 12 ; 15 ; 20\}$ people/min shown in Fig. 4. 

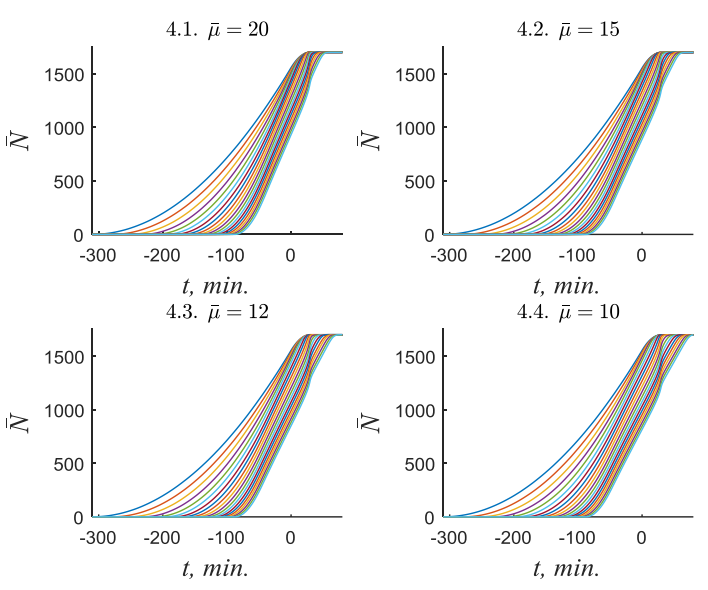

Fig. 4. Dependencies $\bar{N}\left(\lambda_{\max }, \bar{\mu}, t\right)$ (the beginning of the match, $t=0$ ) for different values of parameters $\lambda_{\max }, \bar{\mu}$

Considering to figure 4 :

1. Dependence $\bar{N}\left(T_{1}, T_{2}, \lambda_{\max }, \bar{\mu}, t_{k}\right)$ monotonically increases from zero up to 1700 people in selected range of parameters in any combination. Service of all requests (visitors) ends before time $T_{2}=30 \mathrm{~min}$ for parameters in range given in the Table. 1. For ranges of values of the parameters listed in Table. 2, maintenance of visitors continues after finishing of coming requests in the system.

2. The average number of applications served by the moment $t=0$ is a function:

$$
\bar{N}_{0}=\bar{N}\left(\lambda_{\max }, \bar{\mu}\right)
$$

To calculate the coefficient characterizing the ratio of serviced requests at the moment $t=0$ minutes to the total number of requests serviced (1700) using this function:

$$
P_{0}=\bar{N}_{0} / 1700,
$$

The physical sense of this characteristic for football stadium is fullness of visitors at the beginning of a football match.

Table 1. Parameter values for which service of requests ends before $T_{2}=30 \mathrm{~min}$.

\begin{tabular}{|c|c|c|c|c|}
\hline $\bar{\mu}$, people/min & 20 & 15 & 12 & 10 \\
\hline$\lambda_{\max }$, people/min & {$[10,20]$} & {$[10,19]$} & {$[10,17]$} & {$[10,16]$} \\
\hline
\end{tabular}

Table 2. Parameter values for which service of requests continues after $T_{2}=30 \mathrm{~min}$.

\begin{tabular}{|c|c|c|c|c|}
\hline $\bar{\mu}$, people/min & 20 & 15 & 12 & 10 \\
\hline$\lambda_{\max }$, people/min & {$[21,29]$} & {$[20,29]$} & {$[18,29]$} & {$[17,29]$} \\
\hline
\end{tabular}

The surface defined by the function $\bar{N}_{0}\left(\lambda_{\max }, \bar{\mu}\right)$ shown in Fig. 5.

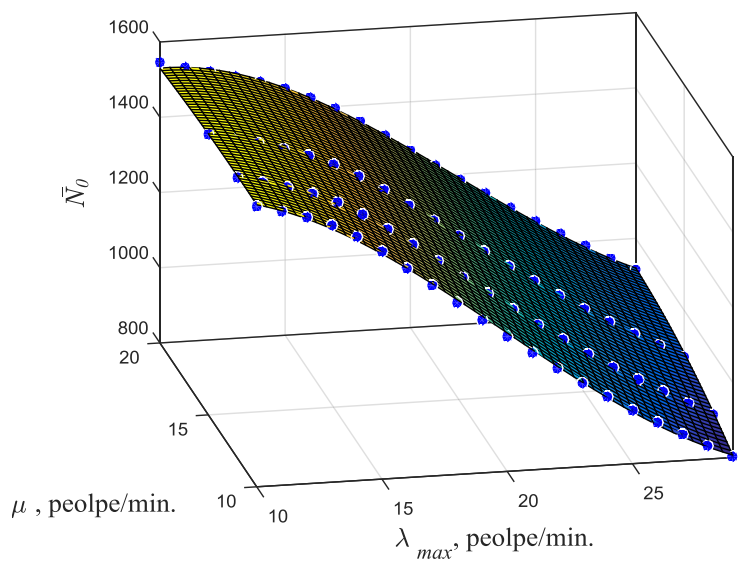

Fig. 5. Function $\bar{N}_{0}\left(\lambda_{\max }, \bar{\mu}\right)$ graph

Figure 5 shows that dependencies $\bar{N}_{0}\left(\lambda_{\max }, \bar{\mu}\right)$, $\bar{P}_{0}\left(\lambda_{\max }, \bar{\mu}\right)$ can be approximated by functions of the form:

$$
\begin{aligned}
\bar{F}_{0}\left(\lambda_{\text {max }}, \bar{\mu}\right)= & a_{0}+a_{1} \lambda_{\text {max }}+a_{2} \bar{\mu}+a_{5} \bar{\mu}^{2}+a_{4} \lambda_{\text {max }} \bar{\mu}+ \\
& a_{3} \lambda_{\text {max }}^{2}+a_{6} \lambda_{\text {max }}^{3}+a_{7} \lambda_{\text {max }}^{2} \bar{\mu}+a_{8} \lambda_{\text {max }} \bar{\mu}^{2}
\end{aligned},
$$

Respective coefficient values $a_{i}, i=\overline{0,8}$, are calculated using the method of least squares. Coefficient values are presented in Table 3.

Table 3. The values of the coefficients of functional dependencies $\bar{N}_{0}\left(\lambda_{\max }, \bar{\mu}\right)$ and $P_{0}\left(\lambda_{\max }, \bar{\mu}\right)$

\begin{tabular}{|c|c|c|}
\hline & $\bar{N}_{0}\left(\lambda_{\max }, \bar{\mu}\right)$ & $P_{0}\left(\lambda_{\max }, \bar{\mu}\right)$ \\
\hline$a_{0}$ & $1570 \pm 110$ & $93 \pm 6$ \\
\hline$a_{1}$ & $41 \pm 11$ & $2,4 \pm 0,6$ \\
\hline$a_{2}$ & $-28 \pm 12$ & $-1,7 \pm 0,7$ \\
\hline$a_{3}$ & $-5,8 \pm 0,5$ & $-0,34 \pm 0,03$ \\
\hline$a_{4}$ & $4,1 \pm 0,7$ & $0,24 \pm 0,04$ \\
\hline$a_{5}$ & $0,1 \pm 0,4$ & $0,003 \pm 0,023$ \\
\hline$a_{6}$ & $0,106 \pm 0,008$ & $0,0062 \pm 0,0004$ \\
\hline$a_{7}$ & $-0,061 \pm 0,011$ & $-0,0036 \pm 0,0006$ \\
\hline$a_{8}$ & $-0,033 \pm 0,020$ & $-0,0020 \pm 0,0011$ \\
\hline
\end{tabular}

The sections of the functions $\bar{N}_{0}\left(\lambda_{\max }, \bar{\mu}\right)$ are dependencies $\quad \bar{N}_{0}\left(\lambda_{\max }, \bar{\mu}_{k}=\right.$ const $), \quad$ there $k=\overline{1,4}$, $\bar{\mu}_{1}=20, \bar{\mu}_{2}=15, \bar{\mu}_{3}=12, \bar{\mu}_{4}=10$, shown in Figure 6 .

Fig. 6 shows:

1. When $\lambda_{\max }=10$ people/min values of the function $\bar{N}_{0}\left(\lambda_{\max }, \bar{\mu}\right)$ are the same for each of the used values of $\bar{\mu}$

2. When $\lambda_{\max }=15 \mathrm{people} / \mathrm{min}$ values of the functions $\bar{N}_{0}\left(\lambda_{\max }, 10\right), \bar{N}_{0}\left(\lambda_{\max }, 20\right)$ differ from each other by $3.67 \%$. 
3. When $\lambda_{\max }=20 \mathrm{people} / \mathrm{min}$ values of the functions $\bar{N}_{0}\left(\lambda_{\max }, 10\right), \bar{N}_{0}\left(\lambda_{\max }, 20\right)$ differ from each other by $9.79 \%$.

4. When $\lambda_{\max }=25 \mathrm{people} / \mathrm{min}$ values of the functions $\bar{N}_{0}\left(\lambda_{\max }, 10\right), \bar{N}_{0}\left(\lambda_{\max }, 20\right)$ differ from each other by $12.86 \%$.
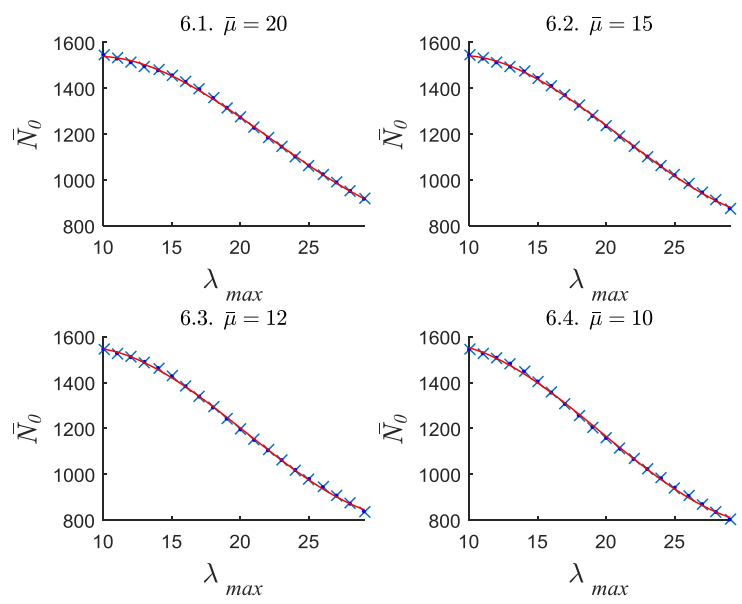

Fig. 6. Dependencies $\bar{N}_{0}\left(\lambda_{\max }, \bar{\mu}=\right.$ const $)$

Thus, in the considered range of values filling of stadium at the beginning of the match will be increase by no more than $12.86 \%$ after doubling the average service rate of a server and respectively a proportional increase of its cost.

\section{Conclusion}

Results of the study suggest the possibility of describing the characteristics of the selected studied type of NQS (ratio of the number of serviced requests for the selected point in time to the total number of serviced requests) using a polynomial that depends on two variables: the maximum arrival rate and the average service rate. The obtained dependence can be used in the design phase of information systems checkpoints (ISCP) objects of mass events, in choosing specifications ISCP turnstiles. The designer is free from the need for simulation modeling NQS. Analysis of other quantitative characteristics studied NQS is the subject of subsequent publications.

\section{References}

1. B.V. Gnedenko, I.N. Kovalenko, Introduction to queuing theory (Moscow, Nauka, 1966)

2. D.G. Kendall, The Annals of Mathematical Statistics, 24, 338. (1953)

3. S.V. Porshnev, I.A. Korelin, D.A. Jakob, 26th International Crimean Conference "Microwave and Telecommunication Technology" (CriMiCo), Moscow, Minsk, 4 (2016)

4. S.V. Porshnev, D.A. Jakob, The study of the functioning of information systems checkpoints objects of mass events (Ekaterinburg, Institut jekonomiki UrO RAN Publ., 2014)

5. S.V. Porshnev, Mathematical models of information flows in the backbone high-speed Internet channels (Moscow, Gorjachaja linija-Telekom Publ., 2015)

6. N.U. Prabhu, Yixin Zhu, Queueing systems, 5, 215246 (1989) 